\title{
Cold-Strontium Laser in the Superradiant Crossover Regime
}

\begin{abstract}
Matthew A. Norcia ${ }^{*}$ and James K. Thompson
JILA, NIST, and University of Colorado, 440 UCB, Boulder, Colorado 80309, USA

(Received 23 October 2015; revised manuscript received 11 January 2016; published 9 March 2016)

Today's narrowest linewidth lasers are limited by mirror motion in the reference optical resonator used to stabilize the laser's frequency. Recent proposals suggest that superradiant lasers based on narrow dipoleforbidden transitions in cold alkaline earth atoms could offer a way around this limitation. Such lasers operating on transitions with linewidth of order $\mathrm{mHz}$ are predicted to achieve output spectra orders of magnitude narrower than any currently existing laser. As a step towards this goal, we demonstrate and study a laser based on the $7.5-\mathrm{kHz}$ linewidth dipole-forbidden ${ }^{3} P_{1}$ to ${ }^{1} S_{0}$ transition in laser-cooled and tightly confined ${ }^{88} \mathrm{Sr}$. We can operate this laser in the bad-cavity or superradiant regime, where coherence is primarily stored in the atoms, or continuously tune to the more conventional good-cavity regime, where coherence is primarily stored in the light field. We show that the cold-atom gain medium can be repumped to achieve quasi-steady-state lasing. We also demonstrate up to an order of magnitude suppression in the sensitivity of laser frequency to changes in cavity length, verifying a key feature of the proposed narrow linewidth lasers.
\end{abstract}

DOI: 10.1103/PhysRevX.6.011025

The ongoing quest for lasers with stable and narrow frequency spectra has led to many advances both in technology and in our understanding of fundamental physics. Applications include optical clocks [1-4], searches for gravitational waves [5], precision studies of atomic interactions [6], and tests of the predictions of relativity $[7,8]$.

The current state-of-the-art laser technology relies on a broadband gain medium that is frequency stabilized by feedback from a spectrally narrow and stable optical cavity. Deviations in the optical cavity length due to thermal and technical fluctuations are imposed on the laser's frequency by the feedback, and are the primary limitation on the frequency stability of today's most stable lasers [9-11]. An alternative approach is to operate in a bad-cavity, or superradiant, regime, where a narrow-band gain medium is confined within a relatively lossy optical cavity, analogous to maser operation in the microwave domain. In a laser operating in the superradiant regime, the frequency of the emitted light is primarily set by the transition frequency of the gain medium rather than the optical cavity. Recently, it has been proposed that one could operate such a laser based on highly forbidden transitions in cooled and trapped alkaline earth atoms, such as the $\mathrm{mHz}$-wide ${ }^{3} P_{0}$ to ${ }^{1} S_{0}$ transition in ${ }^{87} \mathrm{Sr}$, to achieve a linewidth of $1 \mathrm{mHz}$ or narrower [12-15].

\footnotetext{
* Corresponding author. matthew.norcia@colorado.edu

Published by the American Physical Society under the terms of the Creative Commons Attribution 3.0 License. Further distribution of this work must maintain attribution to the author(s) and the published article's title, journal citation, and DOI.
}

Subject Areas: Atomic and Molecular Physics

Here, we take a step towards realization of such a frequency standard by demonstrating and characterizing lasing on the 7.5-kHz-wide dipole-forbidden ${ }^{3} P_{1}$ to ${ }^{1} S_{0}$ transition at $689 \mathrm{~nm}$ in an ensemble of ${ }^{88} \mathrm{Sr}$ tightly trapped in a 1D optical lattice. This laser operates at the crossover between good- and bad-cavity regimes, and can be tuned between the two by broadening the lasing transition. We demonstrate that this laser can be repumped, resulting in quasi-steady-state operation, and show that the frequency of the emitted light is set primarily by the atomic transition frequency when operated in the bad-cavity regime, and by the optical cavity resonance frequency in the good-cavity regime.

The bad-cavity regime of laser physics has been explored in the optical domain in gas lasers [16], with homogeneous and inhomogeneous transition linewidths of hundreds of $\mathrm{MHz}$, and in a 4-level system in cesium with a Dopplerbroadened gain bandwidth of $9 \mathrm{MHz}$ [17]. In the microwave domain, masers operate deep in the bad-cavity regime [18]. Rare-earth doped solid-state lasers utilize transitions with long-lived excited states, but with inhomogeneous broadening of hundreds of $\mathrm{GHz}$ [19]. Raman dressing has been used to create long-lived virtual states with low inhomogeneous broadening, which have been used to demonstrate key properties of optical lasing very deep into the bad-cavity regime [20-23], and deep into the good-cavity regime [24]. The results presented here explore lasing on a true optical transition in a regime where both the homogeneous and inhomogeous linewidths of the gain medium can be made small compared to the cavity linewidth. This is a key step towards a useful frequency reference based on even narrower transitions [12-14,25,26]. 


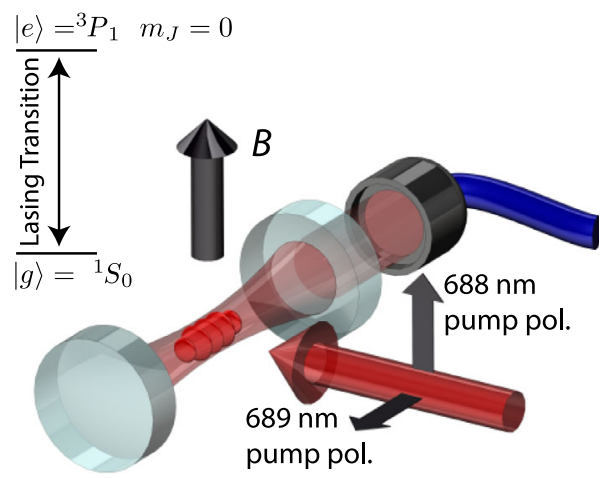

FIG. 1. Energy level and experimental diagrams. The lasing transition is the $7.5-\mathrm{kHz}$ wide ${ }^{3} P_{1}, m_{J}=0$ to ${ }^{1} S_{0}$ transition in ${ }^{88} \mathrm{Sr}$. Atoms are confined by a $1 \mathrm{D}$ optical lattice in a finesse 24000 cavity with linewidth $\kappa=2 \pi \times 160 \mathrm{kHz}$. A magnetic field $B$ is oriented perpendicular to the cavity axis, and pump lasers at 688 and $689 \mathrm{~nm}$ are applied with directions and polarizations as shown. Collectively enhanced emission into the TEM00 mode of the cavity is collected in an optical fiber and sent to one of various detectors.

Our system, also described in Ref. [27], consists of up to $N \sim 100000{ }^{88} \mathrm{Sr}$ atoms cooled to $9 \mu \mathrm{K}$ and confined by an optical lattice within a high-finesse optical cavity (Fig. 1). Atoms are prepared via a two-stage cooling process, with initial capture and cooling using the dipole-allowed ${ }^{1} S_{0}$ to ${ }^{1} P_{1}$ transition at $461 \mathrm{~nm}$, and final cooling and lattice loading using the narrow ${ }^{1} S_{0}$ to ${ }^{3} P_{1}$ transition. The 813-nm lattice is supported by a TEM00 mode of the cavity, which provides intensity buildup for the lattice, and precise spatial registration of the atoms with respect to the cavity mode. A separate spectroscopy signal from the ${ }^{1} S_{0}$ to ${ }^{3} P_{1}, m_{J}=0$ transition allows us to stabilize and precisely tune the cavity resonance frequency.

At the lasing frequency of $689 \mathrm{~nm}$, the cavity has a finesse of 24000 and a linewidth $\kappa=2 \pi \times 160 \mathrm{kHz}$. The lasing occurs on the $|e\rangle \equiv\left|{ }^{3} P_{1}, m_{J}=0\right\rangle$ to $|g\rangle \equiv\left|{ }^{1} S_{0}\right\rangle$ transition at $689 \mathrm{~nm}$, which has a natural decay linewidth $\gamma=2 \pi \times 7.5 \mathrm{kHz}$. The atomic coupling to the cavity mode is inhomogeneous in strength because the lasing and trap wavelengths differ significantly. A single atom trapped at an antinode of the lasing mode exchanges excitations with the TEM00 mode at a frequency $2 g_{0}=2 \pi \times 21.2 \mathrm{kHz}$. Accounting for inhomogeneous coupling, this frequency is collectively enhanced to as much as $\Omega=g_{0} \sqrt{2 N} \sim 2 \pi \times(1-5)$ MHz. Here, $N$ refers to the total number of atoms trapped in the lattice. Our system operates in the single-particle weak-coupling regime, $C=\left(4 g_{0}^{2} / \kappa \gamma\right)=0.41(4)<1$, but in the collective strong coupling regime, $N C \gg 1$.

When many atoms are placed in state $|e\rangle$, the collectively enhanced coupling to the cavity causes the atoms to quickly decay to $|g\rangle$ by emitting a pulse of light into the cavity. We detect this light on an avalanche photodiode, with a single trace shown in Fig. 2(a). The collectively enhanced emission rate exceeds the cavity linewidth, which in turn is much greater than the atomic decay rate; i.e., $\Omega \gg \kappa \gg \gamma$. In this regime, the light pulse is partially reabsorbed by the atoms, and reemitted into the cavity several times before
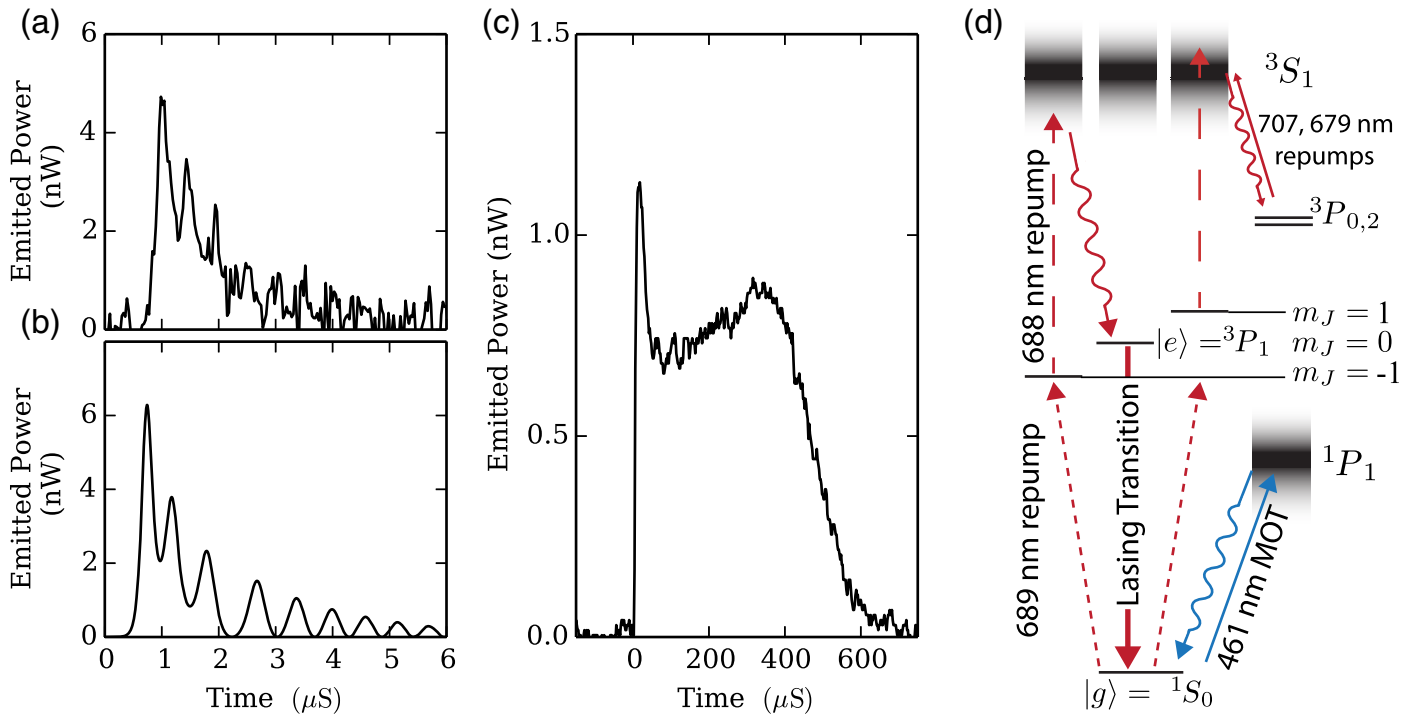

FIG. 2. (a) Laser power emitted from the cavity during a collectively enhanced pulse, with $N=36000$ atoms. The atoms are optically pumped into $|e\rangle$ at $t=0$. (b) Numerical simulation of (a) with no free parameters. (c) The power emitted from the cavity during continuously repumped lasing with $N=60000$ atoms. Each atom emits around 35 photons into the cavity before repump-induced heating causes laser operation to cease. For scale, $1 \mathrm{nW}$ corresponds to 3500 intracavity photons. (d) Repumping scheme. Atoms are incoherently repumped through ${ }^{3} P_{1}, m_{J}=-1$ to ${ }^{3} S_{1}$, where they decay into the ${ }^{3} P$ manifold. Atoms that fall into ${ }^{3} P_{0}$ or ${ }^{3} P_{2}$ are then repumped by additional lasers via ${ }^{3} S_{1}$. For display purposes, ${ }^{3} P_{0,2}$ and ${ }^{1} P_{1}$ states are shown at arbitrary vertical positions. 
escaping the system via transmission through a cavity mirror, resulting in oscillations in output power observed in both the data and simulation of Figs. 2(a) and 2(b). The time scale of these oscillations is of order $2 \pi / \Omega$, but because of inhomogeneous coupling to the cavity, the atoms do not remain in a fully symmetric state. This modifies the period of oscillations and results in incomplete reabsorption of the initially emitted pulse.

By measuring a vacuum Rabi splitting following the pulse, as described in Ref. [27], we infer an atom number of $N=36000$ for the trial shown in Fig. 2(a), up to fluctuations between trials of around $20 \%$. We simulate the pulse dynamics for this atom number by integrating a set of optical Bloch equations [28] that also account for the inhomogeneous coupling to the cavity mode. The results are shown in Fig. 2(b), with good qualitative agreement to observed pulses.

We next apply continuous repumping from $|g\rangle$ back to $|e\rangle$ to operate the laser in a steady-state manner. The repumping process both maintains population inversion and causes decay of the transverse coherence of the atomic ensemble, which reduces the collectively enhanced emission rate. When a steady-state operating condition exists, the total rate of emission from ${ }^{3} P_{1}$ matches the singleparticle rate $w$ at which atoms are optically pumped out of $|g\rangle$. The repumping process also homogeneously broadens the ground state to a width $w$. When operating with $w \ll \kappa$, we access the superradiant regime, where the cavity decay rate exceeds the collectively enhanced emission rate, other damping rates, and the bandwidth of the gain medium.

In Fig. 2(c), we show the laser output power for a representative run of the experiment, with repumping applied to an ensemble of $N \simeq 60000$ atoms initially in $|e\rangle$. We can sustain lasing for up to $1.5 \mathrm{~ms}$, with higher power operation possible for durations of around $500 \mu \mathrm{s}$, as shown. In such a trial, each atom emits up to 35 photons before lasing ceases, most likely as a result of photon recoil heating during the repump process.

In contrast to gas and solid-state lasers that operate on similarly long-lived transitions, our laser also operates in a regime of low inhomogeneous broadening. The frequency scale to which inhomogeneous broadening should be compared is the collectively enhanced emission rate, which in the steady-state case is set by $w[29,30]$. When inhomogeneous broadening is comparable to or less than the collectively enhanced emission rate, the majority of atoms in the ensemble can phase synchronize and contribute to collectively enhanced emission.

In our system, Doppler broadening is suppressed because the atoms are confined to much less than the optical wavelength along the cavity axis. However, the lattice contributes a polarization-dependent shift of the lasing transition [31]. The degree to which this shift is inhomogeneous depends on the temperature of the atoms, which increases during laser operation. At the beginning of laser operation, we estimate inhomogeneous broadening to be less than $30 \mathrm{kHz}$ FWHM for our typical lattice polarization. In principle, this broadening can be tuned to zero. For our range of operating parameters, the majority of atoms have transition frequencies within $w$ of the average transition frequency, allowing them to contribute to lasing.

The repumping mechanism is shown in Fig. 2(d), with beam directions and polarizations shown in Fig. 1(a). A magnetic field is applied perpendicular to the cavity axis, splitting out the $m_{J}= \pm 1$ states by $\pm 7 \mathrm{MHz}$. The 689 -nm repump light is applied near resonance with the ${ }^{1} S_{0}$ to ${ }^{3} P_{1}$, $m_{J}=-1$ transition. Additional $\pi$-polarized 688-nm repump light resonant with the dipole-allowed ${ }^{3} P_{1}$ to ${ }^{3} S_{1}$ transition is applied from the same direction. Because of dipole selection rules, this 688-nm pump couples the ${ }^{3} P_{1} m_{J}= \pm 1$ states to ${ }^{3} S_{1}$, while the $m_{J}=0$ state is unaffected. Single-particle spontaneous emission takes atoms from the ${ }^{3} S_{1}$ states to the ${ }^{3} P$ states, and additional repump lasers at 707 and $679 \mathrm{~nm}$ are applied to depopulate the metastable ${ }^{3} P_{2}$ and ${ }^{3} P_{0}$ states. We emphasize that because atoms reach ${ }^{3} P_{1}$ by single-particle spontaneous decay, we do not expect any coherence between the pump lasers and the emitted light.

We believe the lasing terminates when heating from free-space scattering caused by the repump process reduces the product $N C$ both by causing atom loss and by reducing the coupling of remaining atoms to the cavity. For each lasing photon emitted into the cavity, we estimate roughly 13 photon recoils are imparted during the repump process. For 35 lasing photons per atom, this would contribute heating comparable to the lattice depth.

In order to be useful as a frequency reference, one would like the laser to operate continuously for as long as possible, and the limitations imposed by recoil heating in the repump process must be addressed. It has been proposed that Raman sideband cooling when atoms are in ${ }^{3} P_{2}$ during the repump process could be used to provide cooling and counteract this heating [12]. Laser operation would still be limited by the lifetime of atoms in the lattice, which may be shortened by collisions in a dense ensemble. It may thus be desirable to engineer a constant source of atoms [26], either prepared initially in the excited state or used in conjunction with a repumping scheme similar to the one demonstrated here.

In our laser, output power exhibits a characteristic threshold behavior versus $w$, as shown in Fig. 3(a). In the regime that the total atomic transverse decoherence rate $\gamma_{\perp}=\gamma / 2+w / 2+\gamma_{\perp}^{\prime}$ satisfies $\gamma_{\perp} \ll N C \gamma$, the threshold repumping rate for lasing is simply $w_{t}=\gamma$. This is expected to be true even in the presence of homogeneous or inhomogeneous broadening that exceeds the natural decay rate of the lasing transition [12]. In the above, $\gamma_{\perp}^{\prime}$ accounts for other mechanisms that lead to a decay of atomic coherence. Intuitively, this threshold requirement states 


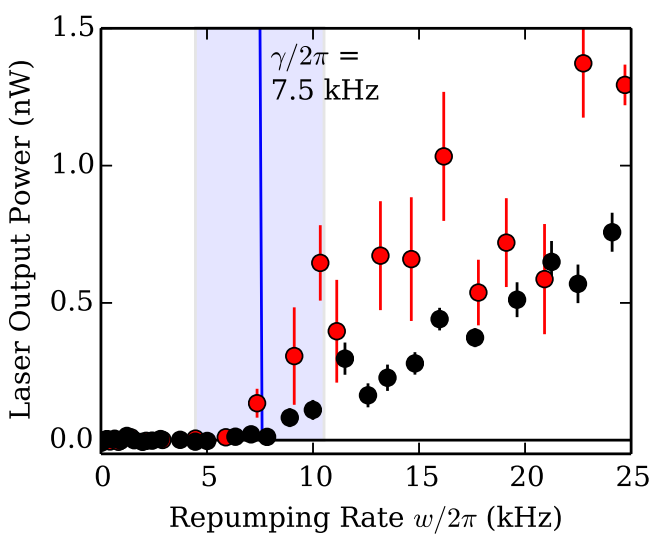

FIG. 3. Threshold behavior of laser. Laser output power in quasisteady state is plotted versus repumping rate $w$ for no added broadening $\gamma_{\perp}^{\prime}=0$ (red points) and added broadening $\left(2 \gamma_{\perp}^{\prime} \simeq 2 \pi \times 3 \mathrm{MHz} \gg \gamma, \kappa\right.$, black points). Atomic decay rate $\gamma$ is displayed as vertical blue line, with shaded blue region representing uncertainty in calibration of $w$. For both conditions, the measured threshold repumping rate $w_{t}$ is consistent with $w_{t}=\gamma$.

that in order to establish population inversion, atoms must be removed from $|g\rangle$ at a rate faster than they can undergo single-particle decay from $|e\rangle$ to $|g\rangle$.

We measure output power from the laser in a window between 20 and $60 \mu \mathrm{s}$ after the beginning of laser operation. We tune $w$ by changing the intensity of the 689-nm repump laser and measure $w_{t}=2 \pi \times 8(3) \mathrm{kHz}$ [32], with the error dominated by the calibration of $w$. This is consistent with the predicted threshold $w_{t}=\gamma=$ $2 \pi \times 7.5 \mathrm{kHz}$.

By inducing Rayleigh scattering on the dipoleallowed $|g\rangle$ to ${ }^{1} P_{1}$ transition at $461 \mathrm{~nm}$, we add additional homogeneous broadening $2 \gamma_{\perp}^{\prime} \simeq 2 \pi \times 3 \mathrm{MHz} \gg \kappa, \gamma$. Despite this high scattering rate, we measure the same threshold value of $w_{t}=2 \pi \times 8(3) \mathrm{kHz}$, again consistent with the prediction. By rotating the lattice polarization, we can introduce inhomogeneous broadening that can be varied from less than $30 \mathrm{kHz}$ to as much as $140 \mathrm{kHz}$ FWHM. Between these two conditions, we measure $w_{t}$ to differ by less than $3 \mathrm{kHz}$. This confirms that the dominant factor in determining threshold is indeed the bare atomic decay rate, not the effective transition linewidth.

An important technological appeal of bad-cavity lasers is their reduced sensitivity to fluctuations in cavity resonance frequency. We define the pulling coefficient $P=\Delta f_{\ell} / \Delta f_{c}$, where $\Delta f_{\ell}$ is the change in the lasing frequency created by a change $\Delta f_{c}$ in the cavity resonance frequency. We expect the pulling coefficient to be given by $P=2 \gamma_{\perp} /\left(2 \gamma_{\perp}+\kappa\right)[16,21,23]$. A pulling coefficient $P \ll 1 / 2$ is a key signature that the laser is operating in the bad-cavity, or superradiant, regime with coherence primarily stored in the atoms, not in the light field.

To measure the pulling coefficient, we overlap the laser light emitted from the cavity with a heterodyne beam from a frequency-stabilized 689-nm laser. We change the cavity frequency between trials and compute power spectra from the heterodyne data. From Gaussian fits to these power spectra, we extract the peak frequency of the emitted light. We obtain the pulling coefficient from a linear fit to $f_{\ell}$ versus $f_{c}$, with $f_{c}$ scanned by $1 \mathrm{MHz}$. We repeat this with different values of $w$. When we turn down the repump beams to just above threshold, $w=2 \pi \times 14 \mathrm{kHz}$, we measure a pulling coefficient of $P=0.09(2)$. This indicates that we have reached the bad-cavity regime, where the spectral properties of the emitted light are dominated by the atomic gain medium. When we increase the repump rate such that the effective atomic linewidth becomes comparable to the cavity decay rate, we reach a crossover regime where coherence is shared between atoms and light field and measure a pulling coefficient consistent with $P=1 / 2$.

The rightmost point in Fig. 4(a) is obtained by turning on the 461-nm MOT beams to further increase $\gamma_{\perp}$ by Rayleigh scattering from $|g\rangle$ at a rate $2 \gamma_{\perp}^{\prime} \simeq 2 \pi \times 3 \mathrm{MHz}$. With
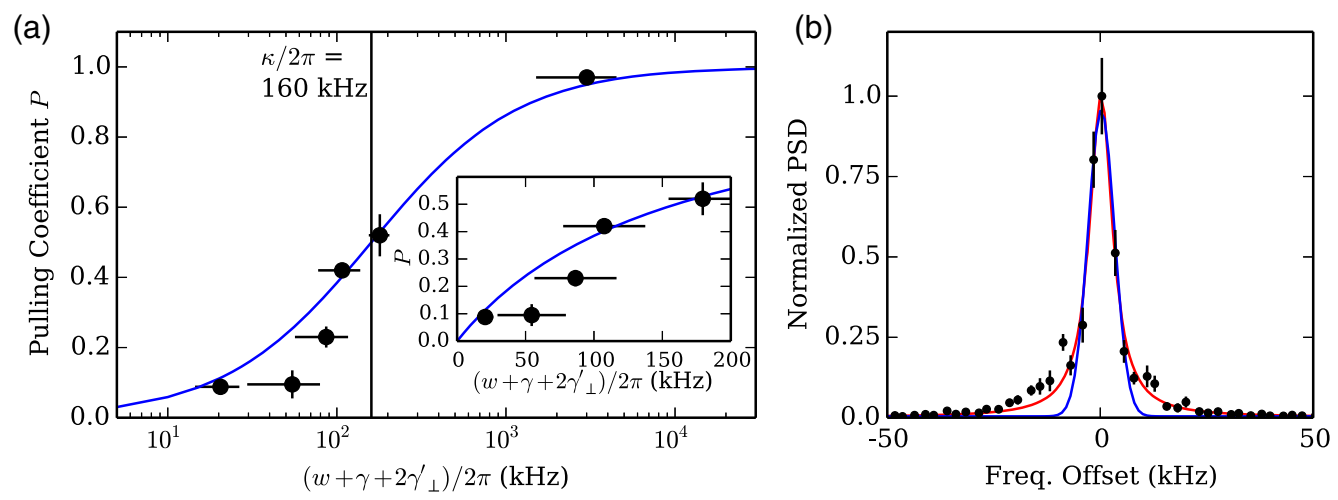

FIG. 4. (a) Pulling coefficient $P$ versus the total homogeneous broadening of the lasing transition, along with prediction (blue). The vertical line at $160 \mathrm{kHz}$ represents the cavity decay rate $\kappa$. The inset displays the left five points on a linear scale. (b) Averaged heterodyne power spectral density (PSD) between output light and 689-nm pump laser, with recentering of each individual trial before averaging. Lorentzian (Gaussian) fits, shown as red (blue) lines, indicate FWHM linewidths of 6.0(3) [4.7(3)] kHz. 
this scattering rate, we measure a pulling coefficient of $P=0.97(3)$. In this highly broadened regime, scattering from the 461-nm MOT beams removes coherence between ${ }^{1} S_{0}$ and ${ }^{3} P_{1}$ without directly affecting the population inversion. By removing the phase coherence from the atoms, we access the good-cavity regime, where the spectral properties of the emitted light are set by the optical cavity.

We bound the linewidth of the emitted light by computing an average power spectrum from heterodyne data taken over many trials. From trial to trial, the center frequency of the power spectrum fluctuates due to lowfrequency noise on the heterodyne laser and optical cavity. We align each power spectrum by shifting its frequency axis by the center frequency obtained from a Gaussian fit, which reduces sensitivity to low-frequency noise. We then fit a Lorentzian and Gaussian to points from all recentered spectra simultaneously. From $150-\mu \mathrm{s}-$ long subsets of the time data, we measure a Lorentzian (Gaussian) FWHM linewidth of 6.0(3) [4.7(3)] kHz. We expect that the fundamental quantum linewidth for these operating conditions would be around $1 \mathrm{~Hz}$ [16], far below our current ability to resolve. Our measured linewidth is Fourier limited for shorter subsets, and limited by acoustic noise on the heterodyne laser for longer subsets. However, our measured linewidth is slightly narrower than the natural linewidth of the lasing transition $(7.5 \mathrm{kHz})$, and far narrower than the linewidth imposed by repumping $(\sim 100 \mathrm{kHz})$, exhibiting the frequencynarrowing characteristic of synchronization in a laser.

To extend the concepts demonstrated here to a technologically useful frequency reference, several paths may be available. To reduce the quantum-limited linewidth to the $\mathrm{mHz}$ level, one would need to push deeper into the superradiant regime by using a lower cooperativity cavity and higher atom number, in a system similar to Ref. [33]. Challenges in implementing this approach would include extending the operation time of the laser, as discussed above, and minimizing light shifts of the lasing transition frequency associated with the repumping process.

We believe another promising approach for an active frequency reference is to operate instead on the ${ }^{1} S_{0}$ to ${ }^{3} P_{0}$ transition in either ${ }^{87} \mathrm{Sr}$ or ${ }^{171} \mathrm{Yb}$, which have linewidths of 1 and $7 \mathrm{mHz}$, respectively. A repumping scheme similar to the one demonstrated here should also work to repump such a laser, though the issues with recoil heating discussed above should be addressed, and more repumping frequency components would be required due to the hyperfine structure of ${ }^{3} P_{2}$. With that in mind, we view the extension of the results demonstrated here to even narrower transitions to be a promising path to realize a frequency standard that has linewidth well below the current state of the art and greatly reduced sensitivity to environmental vibration noise.

We acknowledge contributions to the experimental apparatus by Matthew Winchester, and thank Kevin Cox,
Jan Thomsen, Murray Holland, and David Tieri for useful discussions. The authors acknowledge financial support from DARPA QuASAR, ARO, NSF PFC, and NIST. This work is supported by the National Science Foundation under Grant No. 1125844.

[1] C. W. Chou, D. B. Hume, J. C. J. Koelemeij, D. J. Wineland, and T. Rosenband, Frequency Comparison of Two HighAccuracy Al+ Optical Clocks, Phys. Rev. Lett. 104, 070802 (2010).

[2] N. Hinkley, J. A. Sherman, N. B. Phillips, M. Schioppo, N. D. Lemke, K. Beloy, M. Pizzocaro, C. W. Oates, and A. D. Ludlow, An Atomic Clock with $10^{-18}$ Instability, Science 341, 1215 (2013).

[3] B. J. Bloom, T. L. Nicholson, J. R. Williams, S. L. Campbell, M. Bishof, X. Zhang, W. Zhang, S. L. Bromley, and J. Ye, An Optical Lattice Clock with Accuracy and Stability at the $10^{18}$ Level, Nature (London) 506, 71 (2014).

[4] I. Ushijima, M. Takamoto, M. Das, T. Ohkubo, and H. Katori, Cryogenic Optical Lattice Clocks, Nat. Photonics 9 , 185 (2015).

[5] B. P. Abbott, R. Abbott, R. Adhikari, P. Ajith, B. Allen, G. Allen, R. S. Amin, S. B. Anderson, W. G. Anderson, M. A. Arain et al., LIGO: The Laser Interferometer Gravitational-Wave Observatory, Rep. Prog. Phys. 72, 076901 (2009).

[6] X. Zhang, M. Bishof, S. L. Bromley, C. V. Kraus, M. S. Safronova, P. Zoller, A. M. Rey, and J. Ye, Spectroscopic Observation of SU(N)-Symmetric Interactions in Sr Orbital Magnetism, Science 345, 1467 (2014).

[7] C. Chou, D. B. Hume, T. Rosenband, and D. J. Wineland, Optical Clocks and Relativity, Science 329, 1630 (2010).

[8] C. H. Eisele, A. Y. Nevsky, and S. Schiller, Laboratory Test of the Isotropy of Light Propagation at the $10^{-17}$ Level, Phys. Rev. Lett. 103, 090401 (2009).

[9] T. Kessler, C. Hagemann, C. Grebing, T. Legero, U. Sterr, F. Riehle, M. J. Martin, L. Chen, and J. Ye, A Sub-40-mHzLinewidth Laser Based on a Silicon Single-Crystal Optical Cavity, Nat. Photonics 6, 687 (2012).

[10] K. Numata, A. Kemery, and J. Camp, Thermal-Noise Limit in the Frequency Stabilization of Lasers with Rigid Cavities, Phys. Rev. Lett. 93, 250602 (2004).

[11] M. Notcutt, L. S. Ma, A. D. Ludlow, S. M. Foreman, J. Ye, and J. L. Hall, Contribution of Thermal Noise to Frequency Stability of Rigid Optical Cavity via Hertz-Linewidth Lasers, Phys. Rev. A 73, 031804 (2006).

[12] D. Meiser, J. Ye, D. R. Carlson, and M. J. Holland, Prospects for a MilliHertz-Linewidth Laser, Phys. Rev. Lett. 102, 163601 (2009).

[13] D. Meiser and M. J. Holland, Steady-State Superradiance with Alkaline-Earth-Metal Atoms, Phys. Rev. A 81, 033847 (2010).

[14] J. Chen, Active Optical Clock, Chin. Sci. Bull. 54, 348 (2009).

[15] F. Haake, M. I. Kolobov, C. Fabre, E. Giacobino, and S. Reynaud, Superradiant Laser, Phys. Rev. Lett. 71, 995 (1993). 
[16] S. J. M. Kuppens, M. P. van Exter, and J. P. Woerdman, Quantum-Limited Linewidth of a Bad-Cavity Laser, Phys. Rev. Lett. 72, 3815 (1994).

[17] Z. Xu, W. Zhuang, and J. Chen, Lasing and Suppressed Cavity-Pulling Effect of Cesium Active Optical Clock, arXiv:1404.6021.

[18] H. M. Goldenberg, D. Kleppner, and N. F. Ramsey, Atomic Hydrogen Maser, Phys. Rev. Lett. 5, 361 (1960).

[19] A. E. Siegman, Lasers (University Science Books, Sausalito, CA, 1986).

[20] W. Guerin, F. Michaud, and R. Kaiser, Mechanisms for Lasing with Cold Atoms as the Gain Medium, Phys. Rev. Lett. 101, 093002 (2008).

[21] J. G. Bohnet, Z. Chen, J. M. Weiner, D. Meiser, M. J. Holland, and J. K. Thompson, A Steady-State Superradiant Laser with Less than One Intracavity Photon, Nature (London) 484, 78 (2012).

[22] J. G. Bohnet, Z. Chen, J. M. Weiner, K. C. Cox, and J. K. Thompson, Active and Passive Sensing of Collective Atomic Coherence in a Superradiant Laser, Phys. Rev. A 88, 013826 (2013).

[23] J. G. Bohnet, Z. Chen, J. M. Weiner, K. C. Cox, and J. K. Thompson, Relaxation Oscillations, Stability, and Cavity Feedback in a Superradiant Raman Laser, Phys. Rev. Lett. 109, 253602 (2012).

[24] G. Vrijsen, O. Hosten, J. Lee, S. Bernon, and M. A. Kasevich, Raman Lasing with a Cold Atom Gain Medium in a High-Finesse Optical Cavity, Phys. Rev. Lett. 107, 063904 (2011).
[25] T. Maier, S. Kraemer, L. Ostermann, and H. Ritsch, A Superradiant Clock Laser on a Magic Wavelength Optical Lattice, Opt. Express 22, 13269 (2014).

[26] G. A. Kazakov and T. Schumm, in Proceedings of the European Frequency and Time Conference, 2014 (IEEE, New York City, 2014), pp. 411-414.

[27] M. A. Norcia and J. K. Thompson, Strong Coupling on a Forbidden Transition in Strontium and Nondestructive Atom Counting, Phys. Rev. A 93, 023804 (2016).

[28] J. G. Bohnet, Z. Chen, J. M. Weiner, K. C. Cox, and J. K. Thompson, Linear-Response Theory for Superradiant Lasers, Phys. Rev. A 89, 013806 (2014).

[29] J. M. Weiner, K. C. Cox, J. G. Bohnet, and J. K. Thompson, Phase Synchronization between Two Superradiant Lasers, arXiv:1503.06464.

[30] M. Xu, D. A. Tieri, E. C. Fine, J. K. Thompson, and M. J. Holland, Synchronization of Two Ensembles of Atoms, Phys. Rev. Lett. 113, 154101 (2014).

[31] T. Ido and H. Katori, Recoil-Free Spectroscopy of Neutral Sr Atoms in the Lamb-Dicke Regime, Phys. Rev. Lett. 91, 053001 (2003).

[32] Threshold is determined by fitting to output power versus $w$ a function whose value is zero below a threshold value $w_{t}$ and $a\left(w-w_{t}\right)^{b}$ above.

[33] P. G. Westergaard, B. T. R. Christensen, D. Tieri, R. Matin, J. Cooper, M. Holland, J. Ye, and J.W. Thomsen, Observation of Motion-Dependent Nonlinear Dispersion with Narrow-Linewidth Atoms in an Optical Cavity, Phys. Rev. Lett. 114, 093002 (2015). 